\title{
The Meteorological Service Model of Air and Space Forces Using the Fractal Theory
}

\author{
Vladimir V. Mikhailov* and Sergey L. Kirnosov \\ Military Training and Research Center of the Air Force \\ «Air Force Academy ft. Professor N.E. Zhukovsky and Y.A. Gagarin» \\ 54 a Starykh Bol'shevikov Str., Voronezh, 394064, Russia
}

Received 08.07.2015, received in revised form 16.10.2015, accepted 19.02.2016

Air and space forces of Russia capable of performing a wide range of combat aircraft tasks in various weather conditions. This causes considerable changes in views on the place and role of aviation in military conflicts and on the forms, methods of its combat application and service, including meteorological. The high sensitivity of parameters of functioning of aviation systems weather conditions often leads to the impossibility of use of traditional mathematical methods in building management systems under difficult conditions of weather. Therefore, the paper built the model of meteorological support of aviation systems using the basic elements of the theory of fractals and deterministic chaos theory, which allowed to take into account various combinations of diagnostic and prognostic meteorological methods to support decision making under uncertainty weather information, and perform an extent that there is a chaotic functioning of the system as a whole.

Keywords: fractal structure, meteorological uncertainty, deterministic chaos, weather conditions, stability systems, model of control.

Citation: Mikhailov V.V., Kirnosov S.L. The Meteorological service model of air and space forces using the fractal theory, J. Sib. Fed. Univ. Eng. technol., 2016, 9(3), 366-375. DOI: 10.17516/1999-494X-2016-9-3-366-375.

(c) Siberian Federal University. All rights reserved

* Corresponding author E-mail address: VladimirMihailov36@gmail.com 


\title{
Модель метеорологического обеспечения \\ воздушно-космических сил \\ с использованием элементов теории фракталов
}

\author{
В.В. Михайлов, С.Л. Кирносов \\ ВУНЦ ВВС «ВВА им. проф. Н.Е. Жуковского и Ю.А. Гагарина» \\ Россия, 371600, Воронеж, ул. Старых Большевиков, $54 a$
}

\begin{abstract}
Воздушно-космические силь России способны выполнять широкий спектр боевых авиачионных задач в различных метеорологических условиях. Это вызывает значительные изменения во взглядах на роль и место авиации в военных конфликтах, а также на формы, способы ее боевого применения и обеспечения, в том числе и метеорологического. Высокая чувствительность параметров функиионирования авиационных систем к метеоусловиям зачастую приводит к невозможности использования традиционных математических методов при построении системы управления в сложных условиях погоды. Поэтому в работе построена модель метеорологического обеспечения авиационных систем с использованием основных элементов теории фракталов и теории детерминированного хаоса, что позволило учесть различные сочетания диагностических и прогностических метеорологических способов при поддержке принятия решений в условиях неопределенности метеорологической информации, а также выполнить учет степени хаотичности функционирования всей системы в целом.
\end{abstract}

Ключевые слова: фрактальная структура, метеорологическая неопределенность, детерминированный хаос, условия погоды, устойчивость систем, модель управления.

\section{Введение}

В статье рассматриваются современные военные авиационные системы (BAC), представляющие собой части, соединения и объединения Воздушно-космических сил (ВКС), выполняющие авиационные задачи (АЗ) в условиях неопределенности метеорологической информации (метеонеопределенности) [1-3].

Недавние локальные вооруженные конфликты показали, что современным ВАС, способным выполнять широкий спектр боевых АЗ в различных метеорологических условиях, отводится ключевая роль в ходе проведения войсковых операций. В военных конфликтах ВКС способны проводить крупномасштабные военные действия, решающая роль в которых отводится средствам воздушного нападения, оснащенным элементами высокоточного оружия. Это вызывает значительные изменения во взглядах на роль и место ВАС в военных конфликтах, а также на формы, способы их боевого применения и обеспечения, в том числе и метеорологического.

\section{Материалы и методы}

В трудах отечественных и зарубежных ученых показано, что роль метеообеспечения современных метеозависимых ВАС продолжает оставаться на высоком значимом уровне, так как метеорологические факторы оказывают существенное влияние на эффективность и безопасность их функционирования. Качество современного метеообеспечения ВАС может быть снижено ввиду негативного влияния существующей метеонеопределенности, заключающейся в неидеальности используемой прогностической метеорологической информации

$$
-367-
$$


лицом, принимающим решение (ЛПР), и в отсутствие детерминированных связей характеристик состояния ВАС с условиями погоды, что также приводит к снижению адекватности принимаемых решений на выполнение той или иной АЗ в различных метеорологических условиях [1-3].

Одним из путей снижения степени метеорологической неопределенности является повышение качества прогностической метеоинформации, разработка и распространение которой осуществляется в настоящее время специалистами авиационных метеорологических служб. ЛПР, располагая прогностической метеоинформацией, принимает решение на выполнение АЗ путем сравнения количественных оценок состояния метеоусловий с соответствующими их критическими значениями. Эффективность принимаемых решений, связанных с использованием данного подхода, будет максимальной только в том случае, если ЛПР располагает идеальной прогностической информацией о будущем состоянии погодных условий и знанием точных механизмов воздействия этих условий на функционирование ВАС [1-3]. Однако, как известно, регулярное составление идеальных прогнозов погоды является очень сложной задачей, решение которой вряд ли возможно в обозримом будущем. Кроме того, в условиях боевых действий в отличие от мирного времени могут быть поставлены АЗ особой важности, требующие своего выполнения даже в неприемлемых относительно существущих критических значений погодных условиях (например, эвакуация гражданского населения и раненых, вывод войск из окружения и т.д.). В процессе управления ВАС стратегия ЛПР, подразумевающая принятие положительного решения на выполнение боевой АЗ в условиях повышенной опасности, связанной с метеоусловиями, должна быть оправдана не только важностью АЗ, но и способностью к проведению комплекса мероприятий, направленных на снижение вероятности возникновения аварийной ситуации, связанной с неприемлемыми для авиации погодными условиями. Реализация таких мероприятий может быть осуществима только в том случае, если ЛПР располагает дополнительной специфической информацией об особенностях функционирования ВАС в условиях высокой динамичности метеорологических и других воздействий внешней среды [3]. Данный факт требует исследования новых подходов командира экипажа к построению моделей метеорологического обеспечения ВКС в сложных метеоусловиях и в условиях погоды ниже минимума (минимальных значений высоты нижней границы облаков и дальности видимости, при которых разрешено выполнение взлета, полетного задания и посадки). Одним из таких подходов в статье предлагается подход, связанный с построением модели метеорологического обеспечения ВКС на основе рассмотрения процесса функционирования ВАС с позиций системного подхода. При этом выполнение АЗ представляется в виде целостной структуры - динамической системы «ЛПР - ВАС - метеоусловия». Критерием, по которому оценивается качество принимаемых решений, является максимум вероятности выполнения боевой АЗ по метеоусловиям. Неоднозначность воздействия сложных метеоусловий на механизмы функционирования такой системы приводит к принципиальной нелинейности предполагаемого для использования математического аппарата. Кроме того, высокая чувствительность параметров функционирования динамической системы к начальным условиям приводит к невозможности использования традиционных математических методов при построении модели метеообеспечения ВАС. В таких условиях развитие ситуации в благоприятную либо в другую сторону очень зависит от начальных условий. При этом 
возникает бифуркация (двойственность) вариантов при принятии решений. Поэтому в статье предлагается использование количественных и качественных методов теории фракталов и теории детерминированного хаоса, успешно применяемых в последнее время для анализа нелинейных гиперчувствительных динамических систем [1-4]. Целесообразность применения элементов теории детерминированного хаоса также обусловлена возможностью формализации сложных причинно-следственных связей отдельных элементов динамической системы «ЛПР - ВАС - метеоусловия» [5-8].

Таким образом, целью работы является повышение эффективности функционирования метеозависимых ВАС в процессе их боевого применения в сложных метеоусловиях путем построения модели их метеообеспечения с использованием элементов теории фракталов и детерминированного хаоса.

Методический подход к описанию процесса

функционирования военных авиационных систем

в терминах детерминированного хаоса

Построение модели метеорологического обеспечения ВАС предлагается осуществить в виде разработки совокупности взаимосвязанных моделей и методик поддержки принятия метеозависимых решений на выполнение АЗ при управлении ВАС.

Повышение эффективности и безопасности функционирования современных ВАС возможно, во-первых, при условии снижения степени их метеозависимости и при условии использования ЛПР идеальной прогностической метеоинформации, а во-вторых, построением такого управления и системы поддержки принятия решений на выполнение $\mathrm{A} 3$, которые наилучшим образом учитывали бы имеющуюся неидеальную информацию о погоде. Реализации первого направления в силу ряда экономических, технических и других причин остаются пока не разрешимыми.

В рамках второго направления процесс функционирования современных ВАС целесообразно рассматривать с позиций системного анализа. Такой подход позволит всесторонне изучить особенности построения системы поддержки принятия метеозависимых решений на выполнение АЗ при управлении авиационными подразделениями ВКС.

История развития теории управления неразрывно связана с именами ученых, внесших крупный вклад в эту науку:

1) устойчивость детерминированных систем - И.Л. Вышнеградский (1876 г.), Н.Е. Жуковский (1877 г.), А.М. Ляпунов (1892 г.), А.А. Андронов (1928 г.), Н.М. Крылов (1933 г.), Н.Н. Боголюбов (1933 г.), А.В. Михайлов (1938 г.), А.М. Летов (1945 г.), А.И. Лурье (1945 г.), Л.С. Гольдфарб (1948 г.), С В. Емельянов (1976 г.), Е.Д. Раус (1877 г.), А. Гурвиц (1895 г.), Х. Найквист (1932 г.);

2) динамика и точность стохастических систем - А.А. Марков (1881 г.), А.Я. Хинчин (1936 г.), А.Н. Колмогоров (1936 г.), В.С. Пугачев (1944 г.), И.Е. Казаков (1954 г.), А.Н. Скляревич (1967 г.), Дж. Дуб (1953 г.), В. Феллер (1957 г.);

3) оптимальная обработка информации - К. Шеннон (1948 г.), Н. Винер (1949 г.), Д. Миддлтон (1953 г.), Р. Калман (1960 г.), Р.Л. Стратонович (1966 г.), Я.З. Цыпкин (1968 г.), В.М. Артемьев (1976 г.);

$$
-369-
$$


4) оптимальное управление - Л.С. Понтрягин (1961 г.), А.А. Фельдбаум (1966 г.), Р. Беллман (1960 г.), М. Аоки (1971 г.), А.Е. Брайсон (1972 г.), Дж. Саридис (1980 г.);

5) игровое оптимальное управление - Р. Айзекс (1967 г.), А.Е. Брайсон (1972 г.), Н.Н. Красовский (1970 г.).

В настоящее время необходимость разработки новых качественных и количественных методов исследования динамики систем, построения программных управлений связана с поиском условий устойчивого, надежного и безопасного функционирования сложных динамических систем, имеющих различные особенности.

Отдельный класс задач, связанных с управлением метеозависимыми ВАС, составляют задачи, формулируемые посредством дифференциальных (разностных) уравнений, динамика которых в определенных условиях (при определенных параметрах) демонстрирует различное, в том числе и хаотическое, поведение. При этом, что особенно важно, исходная динамическая система описывается системой детерминированных уравнений. Классическим примером таких систем служит система Лоренца, описывающая конвективные движения в ограниченном объеме [4].

Следует отметить, что к настоящему времени задачи управления в динамических системах, демонстрирующих детерминированный хаос, практически не рассматривались. Если для детерминированных систем методы управления достаточно хорошо разработаны (принцип максимума Понтрягина, принцип оптимальности Беллмана, методы математического программирования), то для стохастических динамических систем, и тем более для систем с детерминированным хаосом, такие методы отсутствуют [1-3].

В различных динамических системах, поведение которых демонстрирует детерминированный хаос, важными являются численные значения управляющих параметров, от которых зависит степень хаотичности системы. Управляя этими параметрами, можно добиться любых (из возможных) состояний динамической системы. На основе этого и предлагается строить управление рассматриваемыми динамическими ВАС, которое будет иметь ряд особенностей.

Главная особенность заключается в том, что для достижения желаемых (эффективных и безопасных) динамических состояний исследуемой авиационной системы построение управления должно основываться на принципе компенсации возмущений. В соответствии с этим принципом управление системой обеспечивает полную компенсацию воздействий внешней среды (метеорологических условий). В таких системах управляющее воздействие поступает в объект управления одновременно с воздействием внешней среды, нейтрализуя его. Однако в открытых системах предусмотреть все возможные возмущения затруднительно. Кроме того, функциональные зависимости между возмущающими и управляющими воздействиями могут быть неизвестны. Поэтому управление динамической ВАС по возмущениям с неполной информацией приводит к накоплению ошибок.

Для минимизации негативного влияния данного факта необходимо проведение качественного анализа уравнений динамики системы с точки зрения установления наличия устойчивых инвариантных множеств (аттракторов), являющихся областями притяжения в фазовом пространстве характеристик функционирования (эволюции) системы. Пусть исследуемая управляемая система описывается системой дифференциальных уравнений:

$$
-370-
$$




$$
\left\{\begin{array}{l}
\frac{\partial X_{1}}{\partial t}=F\left(X_{1}, u_{1}\right) \\
\frac{\partial X_{2}}{\partial t}=F\left(X_{2}, u_{2}\right) \\
\cdots \\
\frac{\partial X_{n}}{\partial t}=F\left(X_{n}, u_{n}\right)
\end{array}\right.
$$

где $\mathbf{X}=\left(X_{1}, X_{2}, . ., X_{n}\right)$ - вектор фазовых переменных, принадлежащий вещественному евклидову пространству $E_{n} ; F$ - нелинейный оператор эволюции динамической системы; $\mathbf{u}=\left(u_{1}, u_{2}, . ., u_{n}\right)$ - вектор управления, принадлежащий множеству вектор-функций $G-$ множеству допустимых управлений.

Основная задача - построение управления, обеспечивающего существование инвариантного множества $M$ системы (1), обладающего требуемыми в приложении свойствами. Если понимать под инвариантным множеством определенный режим функционирования управляемой авиационной системы, то задача управления формулируется как нахождение управления, обеспечивающего устойчивость заданного режима системы. Установление границ инвариантного множества $M$ системы (1) является важнейшей задачей, так как на практике - это множество возможных состояний управляемой системы после стабилизации [4-8].

В классической теории управления достаточно хорошо изучены вопросы динамики управляемых систем с инвариантным множеством. Но в постановке задачи, учитывающей переход режима функционирования системы в состояние детерминированного хаоса, например через серию бифуркаций по сценарию М. Фейгенбаума $[1,3,4]$, ставится вопрос об изучении такой системы в нескольких положениях равновесия, когда множество точек покоя не связно, а сами точки могут быть неустойчивыми.

Исходя из вышесказанного, построение модели управления ВАС, основанной на аттракторах, в настоящее время актуальная проблема. Несмотря на сложность поведения хаотических (странных) аттракторов, знание фазового пространства позволяет представить поведение системы в геометрической форме и, соответственно, предсказать его. И хотя нахождение системы в конкретный момент времени в конкретной точке фазового пространства практически невозможно, область нахождения объекта и его стремление к аттрактору предсказуемы. Ранее такой математический подход в задачах управления не рассматривался.

Другой особенностью построения управления метеозависимой авиационной системой является введение в управляющую систему не только информации о желаемом течении процесса $\mathbf{X}_{u}(t)$, но и информации о реальном его протекании в виде вектора $\mathbf{X}_{r}(t)$, поступающей в управляющую систему по каналу обратной связи. Такое управление замкнутое, и его сущность заключается в коррекции воздействий на управляемый объект с целью перевода его в состояние, необходимое для выполнения АЗ. В этом случае в управляющей системе в любой момент времени может быть измерена ошибка управления

$$
\boldsymbol{\varepsilon}(t)=\mathbf{X}_{u}(t)-\mathbf{X}_{r}(t),
$$


которая используется для формирования управляющего воздействия. Замкнутое управление широко применяется в системах автоматического регулирования, в том числе и для построения систем поддержки принятия метеозависимых решений.

Алгоритм управления в различных ВАС с обратной связью задается функциональной зависимостью управления от выхода или состояния системы. Так, например, в рамках теории детерминированного хаоса динамику численных значений риска, возникающего при выполнении посадки воздушного судна в различных метеоусловиях, предлагается формализовать с помощью рекуррентного соотношения П. Ферхюльста (3), свойства которого демонстрируют универсальность во многих природных и социальных детерминировано-хаотических явлениях [4-6]:

$$
P_{t+1}=k P_{t}\left(1-P_{t}\right)
$$

где $P_{t+1}$ и $P_{t}$ - вероятности возникновения аварийной ситуации в моменты времени $t+1$ и $t$ соответственно; $k$ - управляющий параметр, являющийся синтезированным показателем сложности метеоусловий. Единицы измерения дискретных моментов времени (минуты или секунды) $t, t+1, t+2$ и т.д. в соотношении (3) выбираются в зависимости от требований ЛПР и этапа выполнения посадки воздушного судна с целью адекватного представления текущей системной ситуации.

Анализ аттрактора соотношения (3) показывает, что моделирование вероятности возникновения аварийной ситуации при посадке воздушного судна в диапазоне значений $0 \leq P \leq 1$ возможно только в том случае, если численные значения управляющего параметра $k \in[0,4]$, где $k=0$ соответствует простым метеоусловиям (при этом с течением времени ВАС находится в устойчивом состоянии, $P \rightarrow 0$ ) и $k=4$ - нелетной погоде (при этом $P$ может принимать любое значение от 0 до 1 , что и соответствует состоянию динамического хаоса). Экспериментальные исследования показывают, что количество итераций, необходимое для установившегося движения динамической системы, представленной соотношением (3), - 55-60. Очевидно, что применительно к процессу выполнения посадки воздушного судна $t \approx 60 \mathrm{c}$, в течение которых летчик визуально и по приборам наблюдает метеоусловия посадки, принимает и обрабатывает поток необходимой информации от руководителя полетов, выполняет снижение от четвертого разворота, дальнего приводного радиомаркера и т.д. до точки касания на взлетно-посадочной полосе.

\section{Результаты}

Формальное описание модели метеообеспечения

при управлении военной авиационной системой

Модель управления ВАС представляется в виде множества величин, описывающих процесс ее функционирования и образующих следующие подмножества: совокупность входных воздействий на систему $x_{i} \in X, i=\overline{1, n_{X}}$; совокупность воздействий внешней среды (метеорологических условий) $v_{l} \in V, l=\overline{1, n_{V}}$; совокупность внутренних параметров ВАС $h_{z} \in H, z=\overline{1, n_{H}}$; совокупность выходных характеристик ВАС $y_{j} \in Y, j=\overline{1, n_{Y}}$.

При моделировании ВАС входные воздействия, метеоусловия и внутренние параметры системы являются независимыми (экзогенными) переменными, которые в векторной форме имеют, соответственно, вид

$$
-372-
$$




$$
\begin{aligned}
\vec{x}(t) & =\left(x_{1}(t), x_{2}(t), . ., x_{n X}(t)\right) ; \\
\vec{v}(t) & =\left(v_{1}(t), v_{2}(t), . ., v_{n V}(t)\right) ; \\
\vec{h}(t) & =\left(h_{1}(\Omega, \Xi, t), h_{2}(\Omega, \Xi, t), . ., h_{n H}(\Omega, \Xi, t)\right),
\end{aligned}
$$

где $\Omega$ и $\Xi$ - информация о детерминированных и хаотических свойствах ВАС, а выходные характеристики системы являются зависимыми (эндогенными) переменными и в векторной форме имеют вид

$$
\vec{y}(t)=\left(y_{1}(t), y_{2}(t), . ., y_{n Y}(t)\right) .
$$

Процесс функционирования ВАС описывается во времени оператором $\boldsymbol{F}_{S}$, который в общем случае преобразует экзогенные переменные в эндогенные в соответствии с соотношениями вида

$$
\vec{y}(t)=\boldsymbol{F}_{S}(\vec{x}, \vec{v}, \vec{h}, t) .
$$

Пусть управление ВАС реализуется ЛПР путем принятия решений $x$ на соответствующих этапах ее функционирования исходя из имеющихся временных лимитов $t$, ресурсов $q$ и ожидаемых метеоусловий $v_{l}$. При этом метеорологическая обстановка характеризуется набором метеорологических факторов $\left\{v_{l}\right\}$, пороговое значение которых определяет возможность выполнения боевой авиационной задачи.

Эффективность управления ВАС зависит от качества метеоинформации, от особенностей учета метеорологических условий в процессе поддержки принятия решений, от возможности формализации субъективных факторов, определяющих эффективность использования метеоинформации.

Для обеспечения эффективного функционирования ВАС необходимо повысить качество и ценность метеоинформации. В работе предлагается достичь этого путем построения модели управления ВАС $(A S)$, обеспечивающей достижение оптимума выбранного показателя эффективности функционирования системы - $J(Y(T)$, где $T$ - время функционирования ВАС.

Процесс получения метеоинформации сопровождается применением различных методов или их сочетаний, что приводит к необходимости разработки оптимальной стратегии ее использования.

$$
S^{k}=\left(M_{i}\left(\xi^{\phi}\right), M_{j}\left(\xi^{\text {пр }}\right)\right)^{k}, \forall k=\overline{1, K} ; \forall i=\overline{1, N^{\phi}} ; \forall j=\overline{1, N^{n p}},
$$

где $M_{i}\left(\xi^{\phi}\right)$ и $M_{j}\left(\xi^{\text {пр }}\right)$ - есть $i$-я и $j$-я методики получения фактической и прогностической метеорологической информации соответственно; $k$ - количество стратегий использования метеорологической информации (число элементов множества стратегий $S^{k}$ ); $N^{p}$ и $N^{n p}$ - соответственно количество методик получения фактических и прогностических метеоданных.

Формализовать процесс получения и применения метеорологической информации с учетом информации о степени хаотичности функционирования ВАС предлагается с помощью представленных ниже выражений:

$$
\begin{gathered}
A S=\left\{M(\xi)_{i}, M(\Omega, \Xi)_{i}, M(\mathrm{~S})_{i}, M(Y)_{i}, M(J)_{i}\right\}_{i=1}^{I}, \\
-373-
\end{gathered}
$$




$$
\begin{aligned}
& M(\xi)_{i}=\left\{M_{n p}(\xi)_{i}, M_{\phi}(\xi)_{i}\right\}, \\
& M\left(S^{k}\right)=\left\{M(\xi)_{i}^{k}\right\}_{i=1}^{I I}, \\
& M\left(Y_{j}(T)\right)=f_{j}(q(t), x(t), \xi(t, r)), \forall j=\overline{1, J}, \\
& M(J)_{i}=\left\{q_{i}, x_{i}, M_{n p}(\xi)_{i}, M_{\phi}(\xi)_{i}, M(S)_{i}, M(Y)_{i}\right\},
\end{aligned}
$$

где $M\left(Y_{j}(T)\right.$ - модели состояния ВАС в момент окончания $j$-го временного этапа; $q(t)$ - авиационные ресурсы в момент времени $t ; x(t)$ - оперативно-тактические решения; $\xi(t, r)$ - метеорологические условия, изменяющиеся в пространстве $r$ и во времени $t ; M_{n p}(\xi), M_{\phi}(\xi)$ - модели получения фактической и прогностической метеоинформации; $J$ - количество этапов; $I-$ количество моделей; $S^{k}-k$-я стратегия получения метеоинформации; $M\left(S^{k}\right)$ - модели $k$-й стратегии получения метеоинформации, включающие в себя различное сочетание моделей $M_{n p}(\xi)$; $M_{\phi}(\xi)$ - модели получения информации о состоянии атмосферы; $\Omega, \Xi$ - параметры, определяющие степень устойчивости (хаотичности) функционирования ВАС; $M(J)_{i}-i$-я верификационная модель.

\section{Обсуждение результатов}

В построенной модели метеообеспечения на границе сложных метеоусловий и нелетной погоды переход функционирования ВАС в состояние хаоса подчиняется определенным универсальным законам, которые заключаются в возникновении одной и той же последовательности бифуркаций удвоения периода колебаний (сценарий Фейгенбаума) и в проявлении одних и тех же количественных закономерностей скейлинга. Установленные универсальные закономерности перехода к динамическому хаосу при удвоении периода колебаний были экспериментально подтверждены для широкого класса механических, гидродинамических и других систем (например, переход к турбулентному движению жидкостей и газов).

Применение предложенной модели метеообеспечения ВАС позволяет учесть различные сочетания диагностических и прогностических метеорологических способов при поддержке принятия решений в условиях неопределенности метеорологической информации, а также выполнить учет степени хаотичности функционирования всей ВАС в целом.

Выбор неадекватного управления, приводящего к снижению эффективности функционирования метеозависимой ВАС, связан с наличием остаточной метеонеопределенности, обусловленной в первую очередь характерным для практических ситуаций несовпадением прогностических и фактических метеорологических данных, а также с отсутствием детерминированных законов влияния погоды на выходные параметры ВАС. При формализации влияния метеоусловий на управление ВАС необходимо использовать стратегии получения фактических и прогностических метеоданных.

\section{Выводы}

1. При построении модели метеообеспечения ВКС, реализации которой обеспечивают принятие авиационных управленческих решений, учитывающих скрытые закономерности свойств метеозависимости ВАС, возможно и целесообразно использовать количественные и качественные методы теории фракталов и теории детерминированного хаоса.

$$
-374-
$$


2. Поиск устойчивых и безопасных режимов функционирования ВАС необходимо проводить на основе анализа инвариантных множеств (аттракторов), являющихся областями притяжения в фазовом пространстве характеристик функционирования ВАС.

3. Анализ аттракторов показывает, во-первых, их связь универсальным масштабным подобием, а во-вторых, наличие устойчивых структур - фракталов. Такие структуры гиперчувствительны к внешним воздействиям, что приводит к необходимости их визуализации для дальнейшего использования ЛПР в процессе принятия управленческих решений.

4. Установление границ аттракторов ВАС является важнейшей задачей, так как на практике это множество возможных состояний управляемой системы после стабилизации.

\section{Список литературы}

[1] Михайлов В.В., Семенов М.Е., Кирносов С.Л. // Приборы и системы. Управление, контроль, диагностика, 2015, 1, 12-18. [Mikhaylov V. V., Semenov M. E., Kirnosov S. L.//Devices and systems. Management, control, diagnostics 2015, 1, 12-18.]

[2] Михайлов В.В., Кирносов С.Л. // Наукоемкие технологии, 2015, 4, 10-15. [Mikhaylov V. V., Kirnosov S. L. // High technologies, 2015, 4, 10-15.]

[3] Михайлов В.В., Кирносов С.Л., Гедзенко М.О. // Вестник Воронежского государственного университета. Системный анализ и информационные технологии, 2014, 2, 35-42. [Mikhaylov V. V., Kirnosov S. L., Gedzenko M. O. // Bulletin of the Voronezh state university. System analysis and information technologies, 2014, 2, 35-42.]

[4] Морозов А.Д. Введение в теорию фракталов. Москва-Ижевск: Институт компьютерных исследований, 2004, 160 c. [Introduction to the theory of fractals. Moscow-Izhevsk: Institute of computer researches, 2004, 160 p.]

[5] Михайлов В.В., Кирносов С.Л. // Системы управления и информационные технологии, 2014, 3(57), 85-88. [Mikhaylov V. V., Kirnosov S. L. // Control systems and information technologies, 2014, 3(57), 85-88.]

[6] Михайлов В.В., Кирносов С.Л. // Нелинейный мир, 2014, 3, 14-19 [Mikhaylov V. V., Kirnosov S. L.//Nonlinear world, 2014, 3, 14-19]

[7] Михайлов В.В., Семенов М.Е., Кирносов С.Л. // Нелинейный мир, 2014, 4, 57-63 [Mikhaylov V. V., Semenov M. E., Kirnosov S. L. // Nonlinear world, 2014, 4, 57-63]

[8] Михайлов В.В., Кирносов С.Л. // Системы управления и информационные технологии, 2014, 2(56), 80-84. [Mikhaylov V. V., Kirnosov S. L. // Control systems and information technologies, 2014, 2(56), 80-84.] 